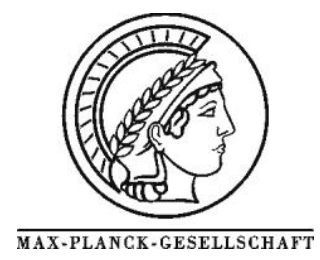

\title{
The crystal structure of $\delta-\mathrm{VOPO}_{4}$ and its relationship to $\omega-\mathrm{VOPO}_{4}$
}

\author{
F. Girgsdies ${ }^{\text {a, }}$, M. Schneider ${ }^{\text {b }}$, A. Brückner ${ }^{\text {b }}$, T. Ressler $^{\text {a,1 }}$, R. Schlögl ${ }^{\text {a }}$ \\ ${ }^{a}$ Department of Inorganic Chemistry, Fritz-Haber-Institut der Max-Planck-Gesellschaft, Faradayweg 4-6, \\ D-14195 Berlin, Germany \\ ${ }^{\mathrm{b}}$ Leibniz-Institut für Katalyse e. V. an der Universität Rostock, Außenstelle Berlin, Richard-Willstätter-Straße 12, \\ D-12489 Berlin, Germany \\ ${ }^{1}$ Current address: Technische Universität Berlin, Institut für Chemie, Sekr. C2, Straße des 17. Juni 135, \\ D-10623 Berlin, Germany. \\ * Corresponding author: e-mail girgsdie@fhi-berlin.mpg.de,
}

Received 19 November 2008; revised 26 February 2009; accepted 24 March 2009; Available online 2 April 2009

\begin{abstract}
The crystal structure of $\delta$ - $\mathrm{VOPO}_{4}$ was determined from powder X-ray diffraction data in the tetragonal space group $P 4_{2} / m b c$ (No. 135) with $a=$ 9.0547(7) $\AA$ and $c=8.6080(8) \AA$. The structure is found to be closely related to that of $\omega$-VOPO $\mathrm{VO}_{4}$, thus disproving two traditional structure hypotheses commonly found in the literature. The structural relationship between the two phases is discussed in the light of a recently observed fast phase transition from $\omega$ - to $\delta$ - $\mathrm{VOPO}_{4}$.
\end{abstract}

Keywords: Vanadium; Phosphate; Crystal structure; Powder diffraction; Ab initio structure determination; Phase transition

\section{Introduction}

The various forms of vanadyl phosphate $\mathrm{VOPO}_{4}$ have attracted the attention of catalysis researchers for more than twenty years [1-12]. More recently, these materials were also investigated in the field of lithium battery research [13-19]. Up to now, seven polymorphs of this compound have been described: $\alpha_{\text {I }}$ [20], $\alpha_{\text {II }}$ [20,21], $\beta$ [22], $\gamma[1,23], \delta[1], \varepsilon[24,25]$, and $\omega[26,27]$. However, compared to the significant interest in these materials, the accumulation of detailed crystal structure knowledge has been rather slow. Structure investigations have been hampered by the fact that several polymorphs are difficult to synthesize as single phase materials of reasonable crystallinity, coupled with the inherent problems of crystal structure solution from powder diffraction data. In particular, the structures of $\gamma$ - and $\delta$ - $\mathrm{VOPO}_{4}$ have defied determination for about two decades, thus leaving room for speculation. Recently, the crystal structure of $\gamma-\mathrm{VOPO}_{4}$ has been solved and reported by Harlow et al. [23]. Here, we describe the determination of the crystal structure of $\delta-\mathrm{VOPO}_{4}$, thus closing another gap in $\mathrm{VOPO}_{4}$ structure research.

\section{Historical background}

The $\gamma$ and $\delta$ phases of $\mathrm{VOPO}_{4}$ were originally described in 1985 by Bordes and Courtine [1]. They investigated the dehydration of $\mathrm{VOHPO}_{4} \cdot 1 / 2 \mathrm{H}_{2} \mathrm{O}$ in order to shed some light on the preparation of $(\mathrm{VO})_{2} \mathrm{P}_{2} \mathrm{O}_{7}$ catalysts for maleic anhydride production. Later, these compounds have variously been reported as minority phases in activated $(\mathrm{VO})_{2} \mathrm{P}_{2} \mathrm{O}_{7}$ catalysts [2-4]. In another 1985 publication, Bordes et al. presented a hypothetical structure model of 
$\gamma-\mathrm{VOPO}_{4}$. The structure was assumed to be related to that of $(\mathrm{VO})_{2} \mathrm{P}_{2} \mathrm{O}_{7}$ in featuring edge-sharing pairs of $\mathrm{VO}_{6}$ octahedra with trans-oriented vanadyl groups [5]. Two years later, Bordes published a comprehensive account on the structure chemistry of V-P-O compounds, including powder XRD data and tentative unit cells for both $\gamma$ - and $\delta$ - $\mathrm{VOPO}_{4}$ [6]. It was proposed that the structure of $\delta$ - $\mathrm{VOPO}_{4}$ would be similar to that of $\gamma-\mathrm{VOPO}_{4}$, but differ in the relative organization of the layers. A more detailed view of the hypothetical $\delta$ - $\mathrm{VOPO}_{4}$ structure can be found in another article published by Bordes in 1988 [7]. These structure hypotheses were based on the supposedly topotactic reactions between $\mathrm{VOHPO}_{4} \cdot 1 / 2 \mathrm{H}_{2} \mathrm{O}$, the $\gamma$ - and $\delta-\mathrm{VOPO}_{4}$ polymorphs, and (VO) $)_{2} \mathrm{P}_{2} \mathrm{O}_{7}$.

A different point of view was taken by Volta and coworkers in 1992 [2]. The indexation of the XRD patterns still followed the work of Bordes. However, the hydration behavior of the various $\mathrm{VOPO}_{4}$ phases, as monitored by in situ laser Raman spectroscopy, gave rise to the assumption that both $\gamma$ - and $\delta$ - $\mathrm{VOPO}_{4}$ should have layered structures related to those of the $\alpha_{\mathrm{I}^{-}}$and $\alpha_{\mathrm{II}}-\mathrm{VOPO}_{4}$ polymorphs. A model of the $\gamma-\mathrm{VOPO}_{4}$ structure was depicted, while details on the hypothetical $\delta-\mathrm{VOPO}_{4}$ structure were published in another article in 1994 [8].

The original unit cell proposals for $\gamma$ - and $\delta-\mathrm{VOPO}_{4}$ were challenged in 1997 by Li et al., who presented new unit cells for both phases based on electron and synchrotron $\mathrm{X}$-ray diffraction [9]. Concerning $\delta$ - $\mathrm{VOPO}_{4}$, however, the unit cells proposed by Bordes (orthorhombic, $a=$

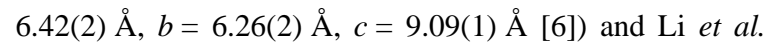
(orthorhombic, $a=8.50 \AA, b=4.67 \AA, c=9.46 \AA$ [9]), respectively, were both rejected in 2000 by Schneider et al., who investigated an exceptionally well crystallized sample with electron and X-ray diffraction [28]. The new unit cell proposal was orthorhombic with $a=9.078(5) \AA, \quad b=$ 9.019(5) $\AA$, $c=8.594(4) \AA$. In contrast, the $\gamma-\mathrm{VOPO}_{4}$ unit cell of Li et al. was finally confirmed in 2005 with the determination of the corresponding crystal structure from combined neutron and synchrotron diffraction data [23]. Unfortunately, the results were published only in the form of a private communication to the ICSD database, i.e. without a corresponding journal article. According to the ICSD entry, the actual crystal structure of $\gamma-\mathrm{VOPO}_{4}$ resembles none of the two traditional structure hypotheses. The structure is complex and indeed peculiar among the $\mathrm{VOPO}_{4}$ polymorphs, since it contains two crystallographically distinct vanadium sites. Furthermore, one of the two vanadium atoms exhibits a truly pyramidal $\mathrm{VO}_{5}$ coordination geometry, as opposed to the distorted octahedral $\mathrm{VO}_{6}$ (or, more precisely, base-capped square pyramidal $\mathrm{VO}_{5+1}$ ) geometry which is usually observed.

Recently, we were able to observe a fast phase transition from $\omega$ - to $\delta$ - $\mathrm{VOPO}_{4}$ [10]. This transformation, together with the overall similarity of the diffraction patterns of both phases, indicated a close structural relationship between the $\delta$ and $\omega$ phases, thus nurturing our interest in solving the $\delta$ - $\mathrm{VOPO}_{4}$ structure. By applying recently de- veloped structure determination techniques in direct space [29] to the X-ray powder diffraction data of Schneider et al. [28], we finally solved the crystal structure of $\delta-\mathrm{VOPO}_{4}$, as will be described below. Similarly to the case of $\gamma-\mathrm{VOPO}_{4}$, the results falsify both of the traditional structure hypotheses found in the literature.

\section{Experimental}

\subsection{Sample preparation and $X$-ray diffraction data collection}

The current study is based on a re-investigation of the diffraction data originally published by Schneider et al. [28]. The sample was prepared from $\mathrm{VOHPO}_{4} \cdot 4 \mathrm{H}_{2} \mathrm{O}$ by heating at $673 \mathrm{~K}$ in flowing air for periods of 5 to 7 days. The diffraction data was collected on a STOE STADI P transmission diffractometer using $\mathrm{CuK} \alpha_{1}$ radiation. Further details on the sample preparation and diffraction experiment can be found in reference [28].

\subsection{Structure determination and refinement}

The program Topas [30] was used for $a b$ initio structure determination from powder diffraction data (SDPD). In the first step, a whole powder pattern decomposition (WPPD) was performed employing the Le Bail method. As a complete assignment of the probable space group(s) based on systematic absences was complicated due to extensive peak overlap, only those tetragonal space groups which showed most obvious incompatibility with the diffraction pattern were deleted a priori from the candidate list. These were all body centered (I) space groups, as well as space groups containing $4_{1}$ or $4_{3}$ as symmetry element. The remaining space groups were subjected to WPPD, which in several cases instantly revealed less obvious violations of systematic absences. Again, these candidates were deleted from the list, leaving several space groups with no or only minor mismatch due to absences. For these remaining space groups, the background, peak profile and lattice parameters were refined in the WPPD and stored for subsequent use in the SDPD attempts.

In a second step, the actual SPDP process was performed in direct space on the diffraction step intensity data [29]. The space groups which were retained after the WPPD screening were tested in no strict order. Background, peak profile and lattice parameters were kept fixed as obtained from the preceding WPPD fit. For every space group, the alternating randomization and refinement process of the structure solution attempt was allowed to run for 10.000 cycles. The results were evaluated with respect to the final $R_{w p}$ value and the resulting model geometry. Promising looking initial models were subjected to further refinement and modified manually if necessary (e.g. to 
Table 1: Crystal and experimental data for $\delta-\mathrm{VOPO}_{4}$

\begin{tabular}{|c|c|}
\hline Chemical formula & $\mathrm{VOPO}_{4}$ \\
\hline Crystal system & tetragonal \\
\hline Space group & $P 4_{2} / m b c$ (No. 135) \\
\hline$a[\AA]$ & $9.0547(7)$ \\
\hline$c[\AA]$ & $8.6080(8)$ \\
\hline$V\left[\AA^{3}\right]$ & $705.74(13)$ \\
\hline$Z$ & 8 \\
\hline$M_{r}\left[\mathrm{~g} \mathrm{~mol}^{-1}\right]$ & 161.91 \\
\hline$\rho_{\text {calc }}\left[\mathrm{g} \mathrm{cm}^{-3}\right]$ & $3.0477(6)$ \\
\hline$\mu\left[\mathrm{cm}^{-1}\right]$ & $271.76(5)$ \\
\hline $2 \theta \min / \max / \operatorname{step}\left[{ }^{\circ}\right]$ & $5 / 95 / 0.02$ \\
\hline$R_{p} / R_{p}{ }^{\mathrm{a}}$ & $0.0199 / 0.1938$ \\
\hline$R_{w p} / R_{w p}{ }^{\prime \mathrm{a}}$ & $0.0267 / 0.1566$ \\
\hline$R_{\exp } / R_{\exp }{ }^{\mathrm{a}}$ & $0.0187 / 0.1096$ \\
\hline$R_{\text {Bragg }} / \mathrm{GOF}$ & $0.0099 / 1.43$ \\
\hline
\end{tabular}

account for the placement of atoms on special positions, which is not automatically done by the program).

The Rietveld refinement of the final crystal structure model, which involved lattice parameters, zero error, profile shape, background, atomic coordinates and a common isotropic temperature factor, was also carried out using Topas [30]. All bond lengths and angles were calculated with Platon for Windows [31,32]. Structure drawings were created with Diamond [33].

\section{Results}

Originally, an orthorhombic unit cell $(a=$

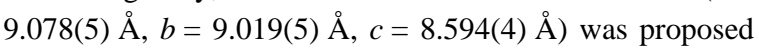
for $\delta-\mathrm{VOPO}_{4}$ [28] based on the diffraction data reinvestigated here. However, considering the similarity of the $a$ and $b$ lattice parameters, we performed WPPD for both orthorhombic and tetragonal symmetry (space groups Pmmm and P4/mmm, respectively). Despite the restrictions imposed by the higher symmetry, the tetragonal fit was not significantly worse than the orthorhombic version. Thus, the higher symmetry was assumed to be more probable.

After space group screening and SDPD attempts as outlined in the experimental section, the space group $\mathrm{P}_{2} b c$ (No. 106) yielded a promising initial solution which was modified manually to place two independent phosphorus atoms on special positions. After further Rietveld refinement, it was found that the resulting structure model was reasonable overall, but the oxygen positions were somewhat unstable, resulting in a significant distortion of the P$\mathrm{O}$ and $\mathrm{V}-\mathrm{O}$ polyhedra. We suspected this to be the result of
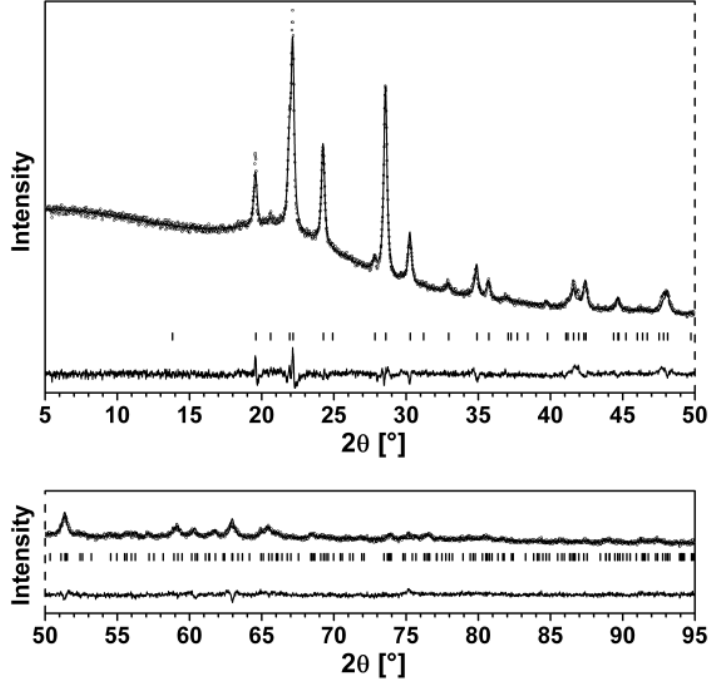

Fig. 1: Comparison of observed (circles) and calculated diffraction pattern (continuous line) of $\delta$ - $\mathrm{VOPO}_{4}$ after final Rietveld refinement. For convenience, the display is split into lower and higher angle range panels (same intensity and angular scale). Calculated peak positions are indicated as tick marks. The continuous line below the tick marks represents the difference between observed and calculated pattern.

using a space group of too low symmetry, as missing symmetry elements typically lead to strong correlations between corresponding parameters of atoms which are actually symmetry equivalent. After analysis of the probable higher symmetry with the program Platon $[31,32]$, the model was transferred into the space group $\mathrm{P}_{2} / \mathrm{mbc}$ (No. $135)$, i.e. effectively adding a horizontal mirror plane. Subsequent refinement of this final model yielded a stable, reasonable geometry. Fig. 1 shows the measured and calculated diffraction pattern, while the final structure data are summarized in Tables 1-3. Further details of the crystal structure investigation may be obtained from Fachinformationszentrum Karlsruhe, 76344 Eggenstein-Leopoldshafen, Germany (fax: (+49)7247-808-666; e-mail: crysdata@fizkarlsruhe.de; http://www.fizkarlsruhe.de/request_for_deposi ted_data.html) on quoting the deposition number CSD-420073.

\section{Discussion}

\subsection{The coordination geometry of vanadium}

Descriptions of the coordination environment of vanadium $(\mathrm{V})$ in oxidic structures usually address the most common geometry as octahedral or (square) pyramidal. In fact, both views are equally appropriate, as they represent two alternative simplifications of the same actual geometry. Typically, the vanadium atom is coordinated by six oxygen atoms at various distances. One of these distances is particularly short and, thus, is commonly interpreted as a $\mathrm{V}=\mathrm{O}$ double bond (vanadyl bond). Four $\mathrm{V}-\mathrm{O}$ bonds of medium 
Table 2: Atomic coordinates for $\delta-\mathrm{VOPO}_{4}$

\begin{tabular}{llllll}
\hline Atom & Site & $x / a$ & $y / b$ & $z / c$ & $\mathrm{U}_{\text {iso }}\left[\AA^{2}\right]$ \\
\hline $\mathrm{V} 1$ & $8 h$ & $0.2478(5)$ & $0.0913(5)$ & 0 & $0.0440(12)^{\mathrm{a}}$ \\
$\mathrm{O} 1$ & $8 h$ & $0.3107(12)$ & $0.2588(14)$ & 0 & $0.0440(12)^{\mathrm{a}}$ \\
$\mathrm{P} 1$ & $4 b$ & 0 & 0 & 0.25 & $0.0440(12)^{\mathrm{a}}$ \\
$\mathrm{O} 2$ & $16 i$ & $0.0973(11)$ & $0.0930(10)$ & $0.1443(12)$ & $0.0440(12)^{\mathrm{a}}$ \\
$\mathrm{P} 2$ & $4 d$ & 0.5 & 0 & 0.25 & $0.0440(12)^{\mathrm{a}}$ \\
$\mathrm{O} 3$ & $16 i$ & $0.3608(11)$ & $-0.0005(7)$ & $0.1497(12)$ & $0.0440(12)^{\mathrm{a}}$
\end{tabular}

${ }^{\mathrm{a}}$ Common temperature factor.

Table 3: Selected bond lengths $[\AA]$ and angles $\left[{ }^{\circ}\right]$ for $\delta-\mathrm{VOPO}_{4}$ with frequencies in parentheses

\begin{tabular}{|c|c|c|c|c|c|}
\hline V1-O1 & $(1 \times)$ & $1.620(13)$ & $\mathrm{V} 1-\mathrm{O} 1^{\mathrm{i}}$ & $(1 \times)$ & $3.057(13)$ \\
\hline $\mathrm{V} 1-\mathrm{O} 2$ & $(2 \times)$ & $1.844(11)$ & V1-O3 & $(2 \times)$ & $1.844(10)$ \\
\hline $\mathrm{P} 1-\mathrm{O} 2$ & $(4 \times)$ & $1.521(10)$ & $\mathrm{P} 2-\mathrm{O} 3$ & $(4 \times)$ & $1.528(10)$ \\
\hline $\mathrm{O} 1-\mathrm{V} 1-\mathrm{O} 2$ & $(2 \times)$ & $104.6(4)$ & O1-V1-O3 & $(2 \times)$ & $103.1(4)$ \\
\hline $\mathrm{O} 1-\mathrm{V} 1-\mathrm{O} 1^{\mathrm{i}}$ & $(1 \times)$ & $169.4(5)$ & $\mathrm{V} 1-\mathrm{O} 1-\mathrm{V} 1^{\mathrm{ii}}$ & $(1 \times)$ & $149.4(6)$ \\
\hline
\end{tabular}

Symmetry codes: ${ }^{\mathrm{i}} 1 / 2-x,-1 / 2+y,-z ;{ }^{\text {ii }} 1 / 2-x, 1 / 2+y,-z$.

length usually have angles around $100^{\circ}$ to the $\mathrm{V}=\mathrm{O}$ bond, resulting in a square pyramid with the vanadyl oxygen atom at the pyramid apex and the vanadium atom displaced slightly from the basal plane towards the center of the pyramid. In addition, a long and highly variable $\mathrm{V} \cdots \mathrm{O}$ distance is commonly found in trans-position to the $\mathrm{V}=\mathrm{O}$ bond, i.e. an additional oxygen atom caps the base of the square pyramid (a notable exception is one of the two vanadium sites found in $\gamma-\mathrm{VOPO}_{4}$ ). Since this sixth distance can be rather long, it may be regarded a weak bond or, alternatively, a short "intermolecular" contact. Thus, depending on the choice whether to accept or reject this sixth distance as being a proper bond, the resulting geometry may be called either (distorted) octahedral or square pyramidal. Verbally, this hybrid geometry may be expressed using the term "base-capped square pyramidal", but a visual representation of such a structure using polyhedra still requires explicit choice between octahedral vs. pyramidal description. Although the octahedral representation looks rather awkward in the case of $\delta$ - $\mathrm{VOPO}_{4}$ because of its very long $\mathrm{V} \cdots \mathrm{O}$ bond, we use this view here to emphasize the relationship between the $\delta$ and $\omega$ polymorphs. In the discussion, however, we will switch freely between octahedral and pyramidal view.

\subsection{The crystal structure of $\delta$-VOPO}

The structure of $\delta-\mathrm{VOPO}_{4}$ contains infinite polar chains of trans-corner sharing $\mathrm{VO}_{6}$ "octahedra" with $[\mathrm{V}=\mathrm{O} \cdots \mathrm{V}=\mathrm{O} \cdots]_{\infty}$ backbone, which run parallel to the [100] and [010] directions. These chains can be rationalized as being organized in "slabs" which are stacked along [001]. The chains lie parallel to each other within a slab, but the orientation between adjacent slabs is perpendicular (Fig. 23). Each $\mathrm{VO}_{6}$ octahedron shares single corners with four different phosphate tetrahedra, and vice versa. Each phosphate group connects four different chains of octahedra, yielding a three-dimensional network. Compared to most other $\mathrm{VOPO}_{4}$ polymorphs, the $\delta$ structure features an unusually long V $\cdots \mathrm{O}$ contact of about $3.1 \AA$. Furthermore, the $\mathrm{O}=\mathrm{V} \cdots \mathrm{O}$ angle of $168^{\circ}$ deviates significantly from the more or less straight arrangement found in other $\mathrm{VOPO}_{4}$ structures. These distortions indicate that the $\delta$ structure is significantly more strained than other $\mathrm{VOPO}_{4}$ polymorphs, which might be an explanation for the reactivity of $\delta$ - $\mathrm{VOPO}_{4}$ with respect to hydration.

If compared to the two traditional structure hypotheses proposed by the groups of E. Bordes [6,7] and J.-C. Volta $[2,8]$, respectively, it is obvious that the actual crystal structure of $\delta$ - $\mathrm{VOPO}_{4}$ differs significantly from the original expectations in several respects. The first model contained pairs of $\mathrm{VO}_{6}$ octahedra which share one common edge, a motif which is found in the structure of $(\mathrm{VO})_{2} \mathrm{P}_{2} \mathrm{O}_{7}$, 

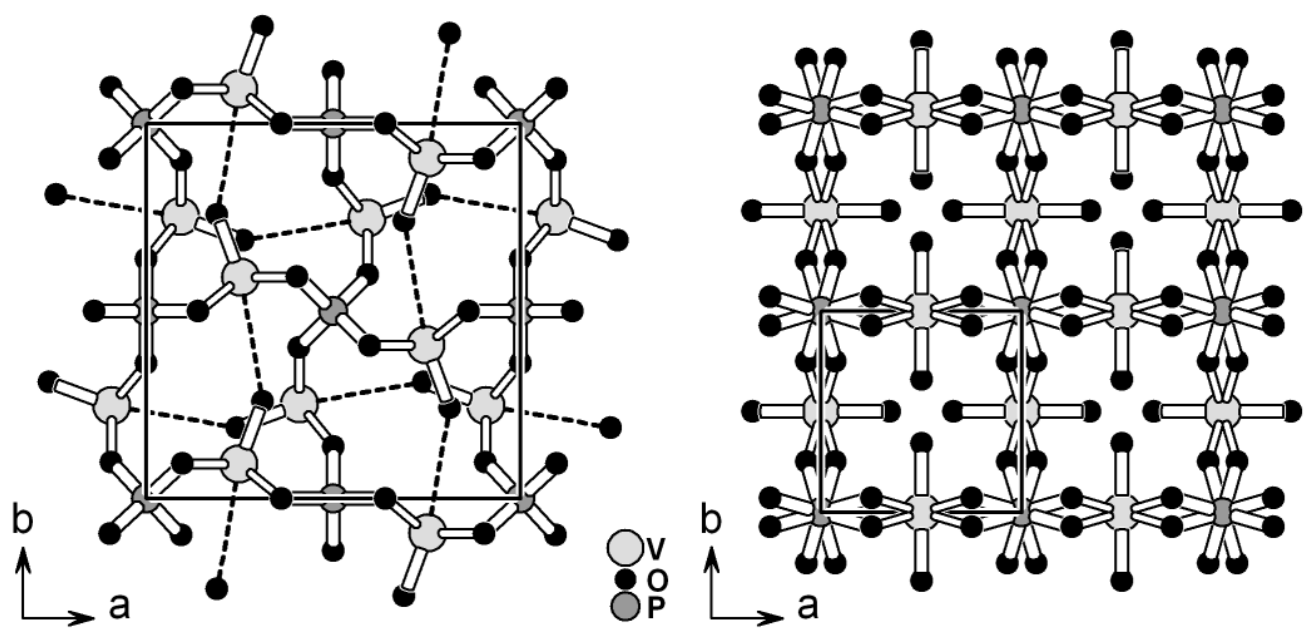

Fig. 2: Crystal structure of $\delta-\mathrm{VOPO}_{4}$ (left), alongside the similar, disordered $\omega$ phase according to Amorós et al. [27] (right), both viewed along the crystallographic $c$ axis. The rather long V $\cdots$ O distances indicated as broken lines in the $\delta$ structure are omitted in the case of the $\omega$ polymorph for clarity, as they would overlap with the disordered $\mathrm{V}=\mathrm{O}$ bonds.

to which both $\gamma$ - and $\delta-\mathrm{VOPO}_{4}$ were supposed to be structurally related. However, it is clear by now that none of the reported actual $\mathrm{VOPO}_{4}$ structures, including the $\gamma$ and $\delta$ phases, exhibits such edge-sharing. The only direct connection between $\mathrm{VO}_{6}$ octahedra observed is corner sharing, which is exclusively realized via the strongly asymmetric vanadyl interaction $\mathrm{V}=\mathrm{O} \cdots \mathrm{V}$, thus leading to infinite chains of trans-corner sharing octahedra. The second model assumes a close relationship with $\alpha_{\mathrm{I}^{-}}$and $\alpha_{\mathrm{II}^{-}} \mathrm{VOPO}_{4}$. Thus, all $[\mathrm{V}=\mathrm{O} \cdots \mathrm{V}=\mathrm{O} \cdots]_{\infty}$ chains were supposed to be parallel to each other, while neglecting the long V $\cdots \mathrm{O}$ interaction would result in a two-dimensional layered lattice. This latter feature enables a rich hydration and intercalation chemistry for $\alpha_{\mathrm{II}}-\mathrm{VOPO}_{4}$, which was the reason for assuming a similar structure for both $\delta$ - and $\gamma-\mathrm{VOPO}_{4}$ because of the relative ease of hydration observed for these phases. The actual crystal structure of $\delta$ - $\mathrm{VOPO}_{4}$, however, includes not only parallel, but also perpendicular orientations of the chains, as detailed above. Furthermore, neglecting the $\mathrm{V}$... O interaction still leaves the three-dimensional nature of the structure intact.

Traditionally, $\gamma$ - and $\delta$ - $\mathrm{VOPO}_{4}$ have been discussed together, and it was usually assumed that they are structurally closely related. While it is apparent now that the two structures are not at all similar to each other, they still have something in common. As already mentioned, both phases show a similar behavior in hydration to $\mathrm{VOPO}_{4} \cdot 2 \mathrm{H}_{2} \mathrm{O}$ $[2,8]$. Structurally, they share a rather low calculated density in comparison to other $\mathrm{VOPO}_{4}$ polymorphs $(3.0(\delta)$ and $2.9(\gamma)$ [23] versus $3.4\left(\alpha_{\text {II }}\right)$ [21], $3.2(\beta)$ [22] and $3.3 \mathrm{~g} / \mathrm{cm}^{3}(\varepsilon)$ [25]). Although $\omega-\mathrm{VOPO}_{4}$ has an even lower density, it is not directly comparable as the crystal structure was determined at elevated temperatures [27]. As the $\mathrm{PO}_{4}$ and $\mathrm{VO}_{5}$ units in $\mathrm{VOPO}_{4}$ can be considered to be relatively rigid, the variable $\mathrm{V} \cdots \mathrm{O}$ distance is one of the main factors contributing to the density variation. In $\delta-\mathrm{VOPO}_{4}$, all $\mathrm{V} \cdots \mathrm{O}$ distances are equivalent and strongly elongated. In $\gamma-\mathrm{VOPO}_{4}$, a similar effect is achieved in a different way: while one of the two different vanadium sites, apart from crystallographic disorder, can be considered having a "normal" V...O bond, the second site has its V ...O distance "stretched to infinity" (i.e. it is absent), leaving the vanadium atom in a truly pyramidal coordination environment. Thus, we may argue that both phases are in an energetically more unfavorable state than e.g. $\beta-\mathrm{VOPO}_{4}$, making them susceptible to hydration despite their three dimensional crystal structures.

\subsection{The relationship between $\delta$ - and $\omega$-VOPO 4}

The recent observation of a fast phase transition from $\omega-\mathrm{VOPO}_{4}$ to $\delta$ - $\mathrm{VOPO}_{4}[10]$ has already indicated a probable close structural relationship between these two polymorphs. Indeed, we find the same general arrangement and connectivity between $\mathrm{VO}_{6}$ octahedra and $\mathrm{PO}_{4}$ tetrahedra. Due to the peculiar disorder of the $\omega-\mathrm{VOPO}_{4}$ structure, which involves both phosphate and vanadyl oxygen positions [27], it cannot be displayed conveniently in a polyhedral fashion. Thus, we use a ball and stick model to depict the disordered $\omega$ structure in Fig. 2, while following Amorós et al. [27] in using an idealized $\omega$ - $\mathrm{VOPO}_{4}$ model with averaged oxygen positions for the polyhedral representation in Fig. 3. The latter clearly shows the close relationship between the two structures, with the unit cell of $\delta$ - $\mathrm{VOPO}_{4}$ corresponding to a quadruple $(2 \times 2 \times 1)$ unit cell of $\omega-\mathrm{VOPO}_{4}$, i.e. $a_{\delta} \approx 2 a_{\omega}$ and $c_{\delta} \approx c_{\omega}$. The doubling of the $a$ lattice parameter in the $\delta$ structure reflects its lower symmetry, as it lacks the vertical mirror planes which are connected to the disorder of the oxygen sites (Fig. 2, right) observed in $\omega-\mathrm{VOPO}_{4}$ (space group $\mathrm{P}_{2} / m m c$ ). Due to this disorder, the $\mathrm{V}=\mathrm{O} \cdots \mathrm{V}=\mathrm{O}$ chains in the $\omega$ phase are straight 

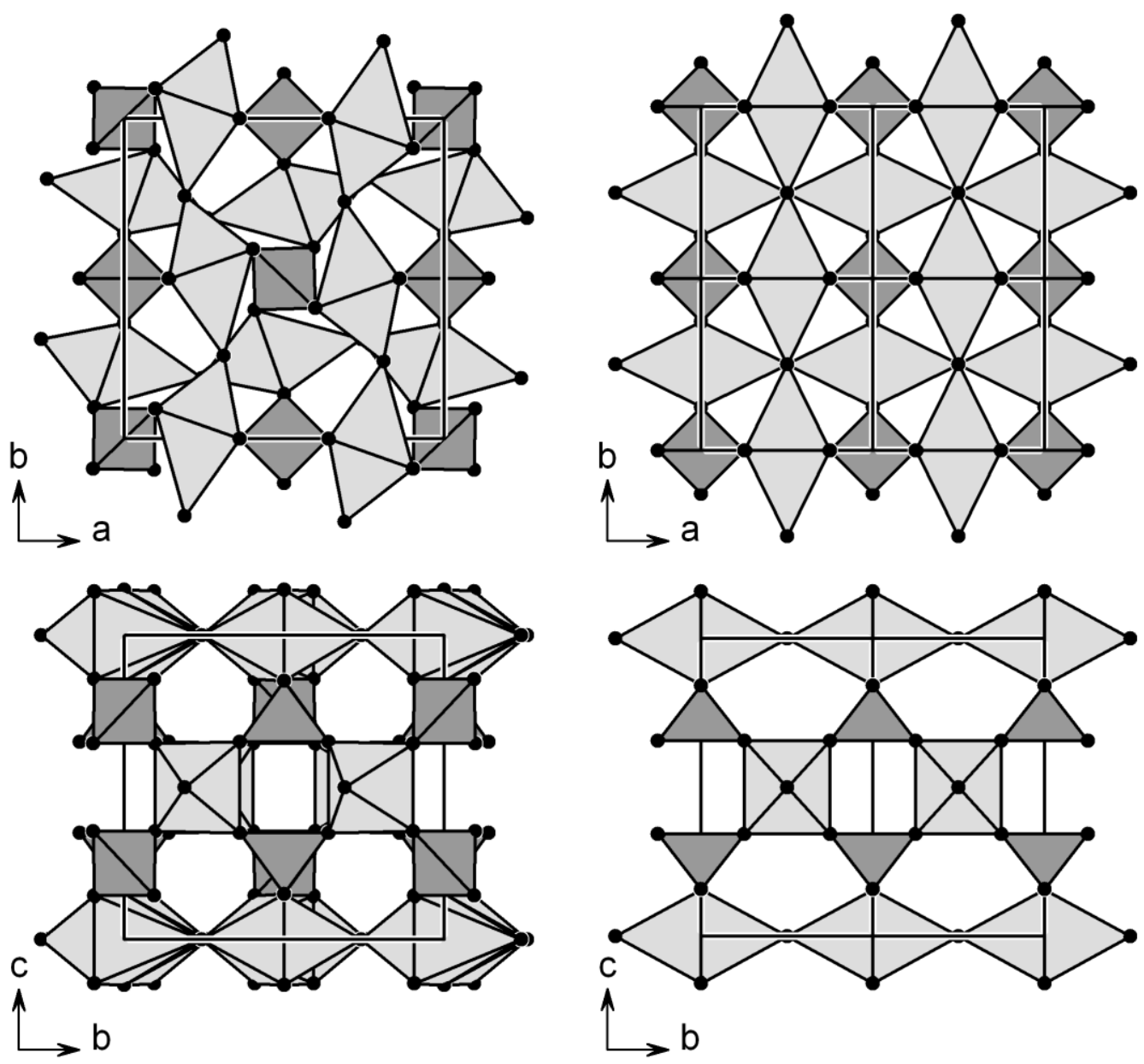

Fig. 3: Comparison of the unit cell of $\delta-\mathrm{VOPO}_{4}$ (left) with a quadruple $(2 \times 2 \times 1)$ unit cell of idealized $\omega-\mathrm{VOPO}_{4}$ (right) in polyhedral view. Both structures are depicted along the crystallographic $c$ (top) and $a$ axes (bottom).

and lack a defined polarity, while $\delta$ - $\mathrm{VOPO}_{4}$ features polar zigzag shaped chains which alter in polarity within a layer. In addition, the $\omega$ structure is expanded significantly in the $a b$ plane relative to $\delta-\mathrm{VOPO}_{4}$, as is apparent from the view along the $c$ axis in Fig. 3.

The nature of the observed phase transition from $\omega-\mathrm{VOPO}_{4}$ to $\delta-\mathrm{VOPO}_{4}[10]$ is still poorly understood. It proceeds within a few minutes at $400{ }^{\circ} \mathrm{C}$ if a small amount (e.g. $1.5 \%$ ) of $n$-butane is added to the gas phase consisting of synthetic air. Thus, the transition is chemically triggered, presumably by slight reduction of the bulk, i.e. introduction of oxygen vacancies. $\omega-\mathrm{VOPO}_{4}$ can be recovered if the $\delta$ - $\mathrm{VOPO}_{4}$ formed is heated to $600{ }^{\circ} \mathrm{C}$ in synthetic air (in absence of $n$-butane). However, as the latter conditions are similar to those used for the original synthesis of $\omega-\mathrm{VOPO}_{4}$ from a structurally unrelated precursor, it seems somewhat questionable whether the inter-conversion of the two polymorphs may be addressed as a "reversible" phase transition. Still, the structural relationship and the speed of the $\omega$-to- $\delta$ transition are very suggestive, hinting that the phase transition might be second order. In order to explore this idea in more detail, it is of importance to realize that for a second order phase transition, a structural relationship which can be expressed via the group-subgroup formalism is manda- tory. With the help of the International Tables for Crystallography [35] it can be shown that such a relationship indeed exists, as the space group of $\delta$ - $\mathrm{VOPO}_{4}, P 4_{2} / \mathrm{mbc}$ (No. 135), is a maximal klassengleiche (isoclass) subgroup of index 2 with respect to the space group $P 4_{2} / \mathrm{mcm}$ (No. 132). The latter, in turn, is again a maximal klassengleiche subgroup of index 2 of $P 4_{2} / m m c$ (No. 131), the space group of the best model for $\omega-\mathrm{VOPO}_{4}$. In both cases, the unit cell of the subgroup (in standard setting) can be derived from the unit cell of its respective supergroup via the transformation $\mathbf{a}^{\prime}=\mathbf{a}-\mathbf{b}, \mathbf{b}^{\prime}=\mathbf{a}+\mathbf{b}, \mathbf{c}^{\prime}=\mathbf{c}$ (vector notation), which doubles the volume of the unit cell in each of the two steps. Thus, the transition from $\omega$ - to $\delta-\mathrm{VOPO}_{4}$ requires in total a fourfold increase in unit cell volume, which is in line with our findings. Furthermore, inspecting the relevant Wyckoff relations (via the intermediate space group $P 4_{2} / \mathrm{mcm}$ ) summarized in Table 4 reveals that the Wyckoff positions experimentally found for $\omega$ - and $\delta$ - $\mathrm{VOPO}_{4}$ match this particular group-subgroup relation. It may seem a contradiction to above statement that not all Wyckoff sites which can be deduced by Wyckoff relations from the $\omega-\mathrm{VOPO}_{4}$ structure are actually observed in 
Table 4: Wyckoff relations [35] for the phase transition from $\omega$ - to $\delta$ - $\mathrm{VOPO}_{4}$ via an intermediate space group (occupancy factors in parentheses, experimentally observed sites in bold)

\begin{tabular}{|c|c|c|c|}
\hline & $\begin{array}{c}\omega-\mathbf{V O P O} \\
P 4_{2} / m m c \\
(\text { No. } 131)\end{array}$ & $\begin{array}{c}\text { [intermediate] } \\
\mathrm{P}_{2} / \mathrm{mcm} \\
\text { (No. 132) }\end{array}$ & $\begin{array}{c}\delta \text {-VOPO } \\
P 4_{2} / m b c \\
(\text { No. } 135)\end{array}$ \\
\hline V & $2 d(1)$ & $4 i(1)$ & $8 h(1)$ \\
\hline $\mathrm{O}_{\text {vanadyl }}$ & $4 k(0.5)$ & $8 n(0.5)$ & $\begin{array}{l}8 \boldsymbol{h}(\mathbf{1}) \\
8 h(0)\end{array}$ \\
\hline $\mathrm{P}$ & $2 e(1)$ & $\begin{array}{l}2 b(1) \\
2 d(1)\end{array}$ & $\begin{array}{l}4 b(1) \\
4 d(1)\end{array}$ \\
\hline $\mathrm{O}_{\text {phosphate }}$ & $16 r(0.5)$ & $\begin{array}{l}16 p(0.5) \\
16 p(0.5)\end{array}$ & $\begin{array}{l}16 i(1) \\
16 i(0) \\
16 i(1) \\
16 i(0)\end{array}$ \\
\hline
\end{tabular}

$\delta$ - $\mathrm{VOPO}_{4}$. However, this is merely a consequence of the disorder in $\omega$ - $\mathrm{VOPO}_{4}$, which makes the transition a disorder-order transition. As typical for such a case, disordered (i.e. partially occupied) sites split into sets of fully occupied and unoccupied sites, the latter of which are naturally not observable in a crystal structure. In summary, we may say that concerning the symmetry relations, the $\omega$-to- $\delta$ transition seems to fulfill the requirements for a second order phase transition. However, as this is only a necessary but not a sufficient condition, it does not provide proof that the transition is indeed second order.

\subsection{Mechanistic aspects of the phase transfor- mation}

While symmetry considerations provide a nice set of tools to explore structural relationships, they are only of limited use when trying to deduce the actual transformation mechanism. Thus, we conducted a thought experiment on this question. For the sake of argumentation, we will now consider the opposite transformation, i.e. from $\delta$ - to $\omega-\mathrm{VOPO}_{4}$, as we can then try to describe the higher symmetry as a special case of the lower one, without complications by disorder. As mentioned before, the structure of $\omega-\mathrm{VOPO}_{4}$ exhibits considerable expansion in the $a b$ plane as compared to $\delta-\mathrm{VOPO}_{4}$. If we regard the phosphate groups and the pyramidal $\mathrm{VO}_{5}$ core of the vanadium coordination sphere as relatively rigid building blocks, it is apparent that the main mechanism of the structure transformation should involve cooperative tilting of the respective polyhedra. In particular, the mechanism should involve the rotation of one of the two phosphate groups, accompanied by a slight shift and tilt of the $\mathrm{VO}_{6}$ octahedra which straightens the chains (Fig. 4). Within the symmetry restrictions of the space group $P 4_{2} / m b c$, only the P1 centered

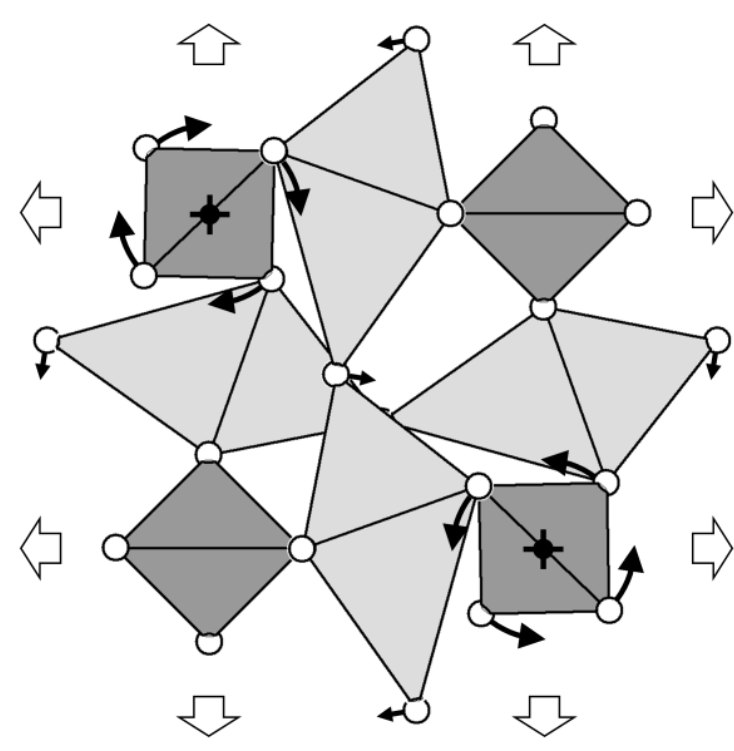

Fig. 4: Schematic representation of one part of the hypothetical $\delta$ to- $\omega$ transition. Small black arrows indicate the proposed atom shifts, i.e. rotation of phosphate groups (rotation axes symbolized by black cross-dots) and the associated straightening of the $[\mathrm{V}=\mathrm{O} \cdots \mathrm{V}=\mathrm{O} \cdots]_{\infty}$ backbone. Thick white arrows represent the resulting lattice expansion.

phosphate group may act as a "hinge", because it is located on a $4 b$ site (symmetry $-4 \ldots$. ). In contrast, the $\mathrm{P} 2$ containing phosphate group is located on a $4 d$ position (symmetry 2. 2 2), in which the horizontal dyads do not permit a rotation without distortion of the tetrahedral shape of the $\mathrm{PO}_{4}$ moiety. The most extreme expansion of the unit cell would be achieved when both phosphate groups finally show a parallel orientation, i.e. when the vanadyl groups are
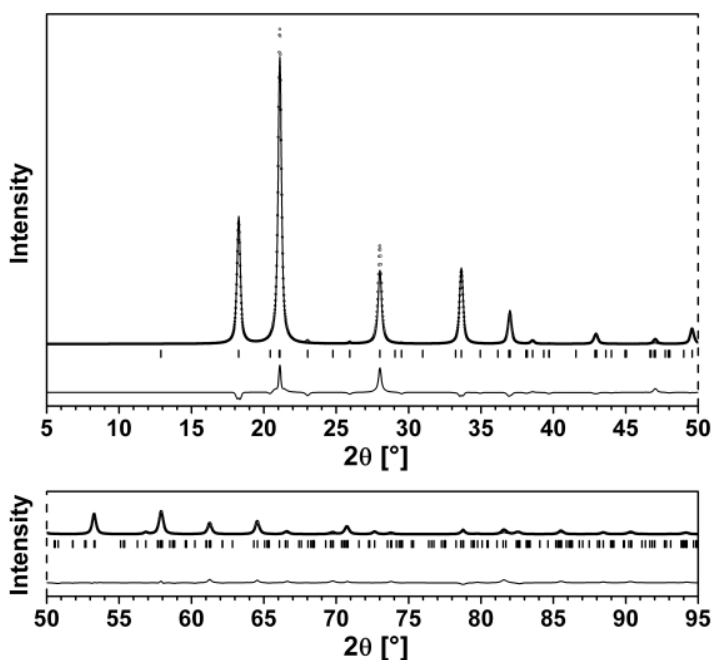

Fig. 5: Calculated diffraction pattern (continuous line) of the $\delta$ - $\mathrm{VOPO}_{4}$ structure modified by Rietveld refinement against "observed" data (circles) simulated from the $\omega-\mathrm{VOPO}_{4}$ structure of Amorós et al. [27]. Tick marks indicate the calculated peak positions. The difference between the two patterns is shown below the tick marks. 

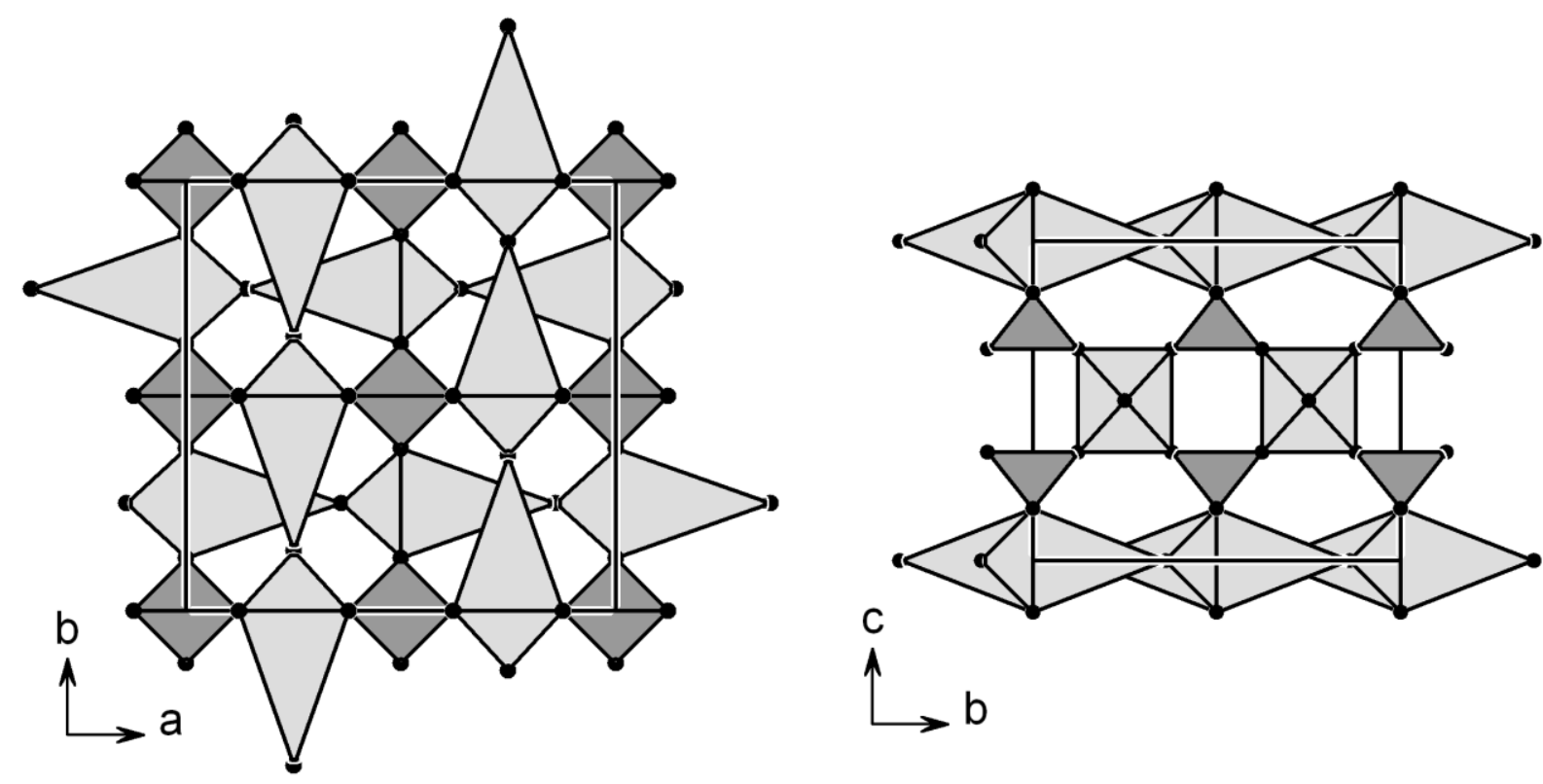

Fig. 6: Structure model of $\delta-\mathrm{VOPO}_{4}$ modified by Rietveld refinement against simulated diffraction data of $\omega-\mathrm{VOPO}_{4}$. View along the crystallographic $c$ (left) and $a$ axes (right).

aligned in straight chains. Apart from the missing disorder, such a modified $\delta$ structure would be quite close to the $\omega$ structure. Thus, in an extension of this thought experiment, we tried to describe the diffraction pattern of $\omega$ - $\mathrm{VOPO}_{4}$ using the $\delta$ structure as a template. Lacking good experimental diffraction data of $\omega$ - $\mathrm{VOPO}_{4}$, we first simulated a diffraction pattern based on the crystal structure model of Amorós et al. [27] to be used as "observed" data. Next, we adjusted the lattice parameters of the $\delta$ - $\mathrm{VOPO}_{4}$ crystal structure to match the $\omega$ pattern and subsequently refined the structure model against the simulated $\omega$ data. The results are shown in Fig. 5 and Fig. 6. Apparently, the crystal structure of $\omega-\mathrm{VOPO}_{4}$ can be approximated reasonably well within the unit cell and space group of the $\delta$ structure. However, this does not mean that we consider this an alternative $\omega$ structure model to the one of Amorós et al. Experimentally, the high-temperature phase $\omega-\mathrm{VOPO}_{4}$ is quite peculiar as it shows a decrease of long-range order upon cooling. Amorós et al. interpret this observation as a consequence of lattice frustration of a structure that is stabilized by dynamical disorder at elevated temperatures [27]. While the crystal structure of $\delta-\mathrm{VOPO}_{4}$ may be successfully adjusted without a change in symmetry up to a point that is very close to the $\omega$ structure, a scenario which would try to explain $\omega$ - $\mathrm{VOPO}_{4}$ solely on the basis of such a modified $\delta$ structure would lack the explanatory power of the Amorós et al. model concerning the the peculiarity of $\omega-\mathrm{VOPO}_{4}$.

\section{Summary and conclusion}

We have shown that the crystal structure of $\delta-\mathrm{VOPO}_{4}$, as determined from powder diffraction data, is related to the structure of the $\omega$ polymorph, which means that it is different from the structure hypotheses proposed in the literature so far. The structural relationship can be described using the group-subgroup formalism, which is a necessary (but not sufficient) condition for the recently observed fast phase transition from the $\omega$ to the $\delta$ phase to be second order. Furthermore, we have explored the possibility of approximating the crystal structure and diffraction pattern of $\omega-\mathrm{VOPO}_{4}$ within the symmetry of the $\delta$ structure. The results of this theoretical experiment show that a cooperative rotation of the relatively rigid $\mathrm{PO}_{4}$ and $\mathrm{VO}_{5}$ polyhedra should be able to modify the $\delta$ crystal structure up to a point that is already very close to $\omega-\mathrm{VOPO}_{4}$. Thus, we conclude that the reverse of this mechanism is most likely part of the experimentally observed phase transformation. In contrast to the rigidity of aforementioned polyhedra, the $\mathrm{V}$... O distance appears to be highly flexible, as indicated by its variability across the different polymorphs of $\mathrm{VOPO}_{4}$. In fact, we believe that the soft yet important $\mathrm{V} \cdots \mathrm{O}$ interaction is a key feature both for the unusual diversity of $\mathrm{VOPO}_{4}$ polymorphs and for the structural dynamics of the $\delta / \omega$ phase couple.

Finally, we would like to emphasize that the successful determination of the $\delta-\mathrm{VOPO}_{4}$ crystal structure is the result of combining experimental data of an exceptional sample with recent developments in structure solution approaches and the ongoing increase in computer power. This demonstrates that in cases where good diffraction data are 
difficult to obtain, it may be well worth revisiting older archived data sets, which could not be fully exploited with the methods available at the time they were measured.

\section{Acknowledgement}

The authors thank G.-U. Wolf for the preparation of the well crystallized $\delta-\mathrm{VOPO}_{4}$ sample, B. Kubias for initiating this fruitful collaboration, and the Deutsche Forschungsgemeinschaft for financial support (SFB 546).

[20] M. Tachez, F. Theobald, E. Bordes, J. Solid State Chem. 40 (1981) 280.

[21] B. Jordan, C. Calvo, Canad. J. Chem. 51 (1973) 2621, ICSD collection code 2889.

[22] R. Gopal, C. Calvo, J. Solid State Chem. 5 (1972) 432, ICSD collection code 9413

[23] R.L. Harlow, Z.G. Li, N. Herron, H.S. Horowitz, E.M. McCarron, J.W. Richardson Jr., B.H. Toby, private communication to the ICSD (2005), ICSD collection code 415213.

[24] S.C. Lim, J.T. Vaughey, W.T.A. Harrison, L.L. Dussac, A.J. Jacobson, J.W. Johnson, Solid State Ionics 84 (1996) 219.

[25] F. Girgsdies, T. Ressler, R. Schlögl, W.-S. Dong, J.K. Bartley, G.J. Hutchings, Solid State Sci. 8 (2006) 807, ICSD collection code 415924.

[26] H. Wibbeke, ICDD Grant-in-Aid (1985), PDF2 pattern 37809.

[27] . Amorós, M.D. Marcos, M. Roca, J. Alamo, A. BeltránPorter, D. Beltrán-Porter, J. Phys. Chem. Solids 62 (2001) 1393.

[28] M. Schneider, M. Pohl, G.-U. Wolf, F. Krumeich, Mater. Sci. Forum 321-324 (2000) 960.

[29] A.A. Coelho, J. Appl. Cryst. 33 (2000) 899.

[30] Topas v2.1, copyright 1999, 2000 Bruker AXS

[31] Platon for Windows Taskbar v1.081, copyright 1995-2005 L.J. Farrugia, University of Glasgow, Glasgow, UK.

[32] PLATON, A Multipurpose Crystallographic Tool, v200905, copyright 1980-2005 A.L. Spek, Utrecht University, Utrecht, The Netherlands.

[33] Diamond v3.1, copyright 1997-2005 Crystal Impact GbR, Bonn, Germany.

[34] R.A. Young, Introduction to the Rietveld method, in: R.A. Young, The Rietveld Method, Oxford University Press, 1993, pp 1-39.

[35] H. Wondratschek, U. Müller, International Tables for Crystallography, Volume A1: Symmetry relations between space groups, Kluwer Academic Publishers, 2004. 